\title{
The Significance of Restoring the Traditional Diet of Chinese People from the High Incidence of Modern Diseases
}

\author{
Bao-song LIU, Meng-fan PENG, Yan-yan MIAO, Ming BAI and \\ Ming-San MIAO ${ }^{a,{ }^{*}}$ \\ Henan University of Traditional Chinese Medicine, Zhengzhou, Henan 450046, China \\ amiaomingsan@163.com \\ ${ }^{*}$ Corresponding author
}

Keywords: Dietary Structure, Eating Habits, Vegetable Food, Animal Food.

\begin{abstract}
From the reform and opening up in 1979 to the year of twenty-first Century, China's economy has been growing rapidly,and great changes have taken place in people's traditional eating habits. Gradually westernized modern diet has become one of the main reasons, which leading the incidence of many chronic diseases increased year by year. In recent years, this article describes the phenomenon of the change of Chinese people's eating habits.It proposed that the country should strengthen scientific guidance, provide policy support, make"healthy diet" as a basic national policy. It also advocated people reasonable collocation diet structure, restored the traditional diet of China.
\end{abstract}

\section{Introduction}

Plant food as the main, animal food as a supplementary dietary structure, following a variety of food nutrition and"the medicine and food homology,diet therapy in medical" treatment constitute a long history, rich cultural connotation Chinese traditional eating habits. Zhong-shan Sun , a pioneer of the democratic revolution said that our modern civilization everything is behind, only the progress of a diet that other countries have not reached. However with the rapid development of China's economy and the invasion of western culture, China's traditional eating habits are facing unprecedented challenges in recent years. From the basic data of the national nutrition survey, the dietary structure of residents in our country entered a rapid transition period since 1980s. The diet before the reform and opening up is a high carbohydrate, low protein, low fat and low vitamin "one high and three low diet". Now the rapid conversion to high energy, high protein, high fat and low dietary fiber "three high and one low diet". The conversion rate of the city is faster than the rural areas obviously especially in 80 s, which is coincided with the significant slowdown in the growth of per capita life expectancy ${ }^{[1]}$. The direct result of modern eating habits is the problem of excess nutrition has become prominent increasingly, followed by obesity, cardiovascular disease, gout and other diseases increased dramatically. Rationality of eating habits and human health are inseparable, only advocate a healthy diet, restore Chinese traditional eating habits,can provide balanced nutrition to the body, promote the body's resistance to disease and maintain the health of the people. 


\section{Traditional Eating Habits of China}

\section{The Structure of Diet}

"We rely on food,without food, without us" .Diet is a manifestation of life activity, the guarantee of health and longevity. The ancient saying that"Only rely on insects and animals, people can not survive. Only by grain that people can thrive". Grain in the diet has an irreplaceable dominant position. "Huangdi Neijing" said grain for the support, fruit for the auxiliary, animal for the benefit, dishe for filling. It reflects the grain (staple food) is the fundamental of survival, fruit, vegetables and meat are staples as the auxiliary, tonic and supplement. This is a good reflection of the traditional Chinese diet.

"Five grains" refers to rice, wheat, millet, corn and beans five kinds of grain crops,we usually call this kind of food cereals. The rice and wheat are the most respected by people in China. Rice can flat stomach and promote muscle growth, wheat can thick intestines, stomach and strong strength. The structure of the human body not only depends on the protein, but also needs sugar as auxiliary materials. The human $60 \%-70 \%$ consumption of energy are provided by the sugar. And the grain contains a lot of starch, it can supply the sugar that human body needs. Cereal is the staple food of the human body, it could account for $50 \%$ in the daily diet.

"Five fruits" is that we now call jujube, chestnut, plum, apricot and peach, refers to all kinds of fruit. They are rich in vitamins, dietary fiber and vegetable protein. The fruit should eaten in raw as far as possible, in order to ensure vitamins are not destructed by cooking. Fresh fruit processed into dried fruits,can make transportation and storage convenience. Despite the loss of water-soluble vitamins, proteins and carbohydrates are increased by dehydration. Fruit can also help digestion after a meal, it is also an adjunct to the balanced diet. The fruit could account for $10 \%$ in the daily diet.

"Five animals" is refers to beef, dog meat, pork, lamb, chicken and other animal foods. The meat contains high protein, high fat, high calorie and essential amino acids. The meat is the most important nutrients to repair tissue and enhance the disease resistance for human body. Because of all kinds of meat is not easy to digest,we can add onions, ginger and garlic in the cooking process to help the stomach better digestion, absorption and can make up for the lack of plant protein. The meat could account for $15 \%$ in the daily diet.

"Five vegetables" refers to all kinds of vegetables. In general, vegetables are as a supplement to the famine in the ancient times. But in modern times, vegetables are indispensable in our dieat.The vegetable can make breath smooth and catharsis easy.The vegetable contains a variety of trace elements, is not only an indispensable supplementary food, but also the main source of dietary fiber. The vegetable could account for $25 \%$ in the daily diet.

\section{The Diet Nutrition}

On the basis of the traditional Chinese diet, pay attention to dietary influence, promote a variety of food and make complementary in the same kind of food. There are more than 2000 kinds of edible plants in China, more than 100 kinds of vegetables, more than 20 kinds of beans and more than 300 kinds of fruits. Many elderly people are mainly based on plant-based food and a variety of daily diet. Diet should also be combined with the four climate, environment and make appropriate adjustments. There are four seasons including warm spring, hot summer, cool autumn, and chill winter .The characteristics of people, human physiology and pathology is easily affected by climate, so the choice 
of food should be adapted to the environment[2]. Of course geography and lifestyle differences also affect the choice of food types to some extent.

\section{The Medicine and Food Homology}

Chinese has a theoretical that the medicine and food homology diet therapy in medical since ancient times. <Huangdi Neijing Taisu> said "When you are hungry, you call it food, while patients with food as medicine", it also reflects the thought of "the medicine and food homology". In TCM nutrition the most basic principle of medicinal using is "dialectical meal" in the prevention and treatment of diseases[3]. There is a clear difference compared to modern nutrition which use a method for delineating nutritional value of food from the analysis of food ingredients and nutrients. Traditional Chinese medicine nutrition has a strong practicality,selecting the appropriate food compatibility. So that all kinds of food corresponding to the declaration, pass, fill, vent, light, heavy, slippery, astringent, dry, wet and other aspects of efficacy.Five flavors of entrance, each have a place to go.We should pay attention to certain corresponding relationship between food born and the five internal organs of the body,so that we can achieve the purpose of disease prevention and treatment[4].

\section{The Dietary Structure Changes of Chinese in Recent Years}

Since the reform and opening up, China's grain planting, processing and supply great changes have taken place with the rapid development of economy. The dietary structure of residents is also changing rapidly, the dietary habits tend to be westernized and the traditional diet based on cereal grains is challenged.

In late July 2005, China made a report about the changes in nutrition and nutritional status of Chinese Residents. The report said that China's dietary structure has changed significantly, the world's per capita meat consumption increased by two times, while China has increased 10 times from 1961 to 2000. In 1992 meat provided 15\% of the energy for the country, while in 2002 was $19 \%$. The average daily consumption of fruit decreased from $98 \mathrm{~g}$ in 1992 to $88 \mathrm{~g}$ in 2002, per capita consumption of vegetables decreased from $319 \mathrm{~g}$ to $252 \mathrm{~g}$, cereal food consumption decreased from 594g in 2002 to 472g in 1992.This dietary habit is far from the per capita recommended by dietary structure Pyramid. We can see that the most prominent changes in the diet is the performance of grain consumption reduced, while fat and high calorie food consumption increased.The traditional rough processing of grain is replaced by large machinery and fine processing, food variety is unitary.The processing is too fine so that the calorific value of staple food increased and huge loss of nutrients. This caused people to excessive intake of high calorie foods, but vitamins, cellulose, trace elements and other nutrients intake inadequately. A survey shows that in the past ten years, the proportion of energy supply of cereal grains in our country has dropped from $67 \%$ to $57 \%$, the proportion of animal food increased from $9 \%$ to $14 \%$ and the ratio of fat increased from $19 \%$ to $28 \%{ }^{[5]}$.

The per capita consumption of animal food has increased year by year.The urban residents per capita consumption of animal food increased from $80 \mathrm{~g}$ to $140 \mathrm{~g}$ from 1989 to 2009 which is significantly higher than the per capita consumption of $75 \mathrm{~g} / \mathrm{d}$ that we advocated. While dietary fiber and mineral intake is obviously insufficient said by Feng-ying Zhai ${ }^{[6]}$. Now people intake of too much energy from animal food coupled with lack of exercise, leading to increased prevalence of obesity easily. At the same 
time diseases related to diet unreasonable, such as hypertension, dyslipidemia, diabetes and other chronic diseases will increase rapidly. Datas show that Chinese residents in patients with hypertension has more than 200 million, increasing more than 3 million cases per year. In 2015 Chinese nutrition and chronic disease status report shows that the overweight rate of 18 years old and above is more than $30 \%$, the obesity rate is more than $10 \%$ and the health situation is worrying in our country. Therefore people's diet structure has become the problem of the whole society.

Improve the nutritional status of residents and balance the diet structure is an important part of the people's livelihood project.The general office of the State Council promulgated the Outline of China Food and Nutrition Development (2014 - 2020). The Outline based on the protection of effective supply of food, optimizing the diet structure, improve nutrition, draw a new blueprint on food and nutrition development until 2020. Such as the national grain output stable at more than 5.5 tons, the average daily energy intake was 2200 to $2300 \mathrm{kcal}$,then the rates of overweight, obesity and dyslipidemia were significantly decreased. By 2020 the per capita consumption of grain is 135 kilograms, 12 kilograms of edible vegetable oil, 13 kilograms of beans, 29 kilograms of meat, 16 kilograms of eggs, 36 kilograms of milk, 18 kilograms of aquatic products, and 140 kilograms of vegetables and 60 kilograms of fruits. The Outline put forward to curb the overweight and obesity of adolescents in cities and towns, guide the reasonable diet structure, improve the people's health level and determine it as one of the important contents of social construction.

\section{The High Incidence of Modern Diseases}

The 2014 Chinese Health and Family Planning Statistics Yearbook datas show that the average incidence rate of hypertension increased about 6 times, the incidence of diabetes increased by nearly 7 times, malignant tumor, stroke, heart disease and other disease incidence increased year by year from 2003 to 2013 in China. In addition to smoking, excessive drinking, air pollution and other reasons, the changes in diet is still an element that can not be underestimated. The developed countries such as Europe and the United States as a representative of high energy, high fat, high protein and low fibe that so-called "three high and one low" diet, it is prone to excess nutrition. A large number of studies have shown that excess nutrition is one of the main factors of obesity, cardiovascular disease, diabetes ,cancer and other chronic diseases which is an important reason for many scholars called for the prevention of food Westernization ${ }^{[7]}$.

Nutrition excess damage the health of Westerners seriously. In 1986 more than 5\% of doctors in the United States did not treat patients with dietary therapy. In the past 20 years, the level of TC in the population of the United States has decreased which is related to the degree of attention of the doctor and the patient ${ }^{[8]}$.A research made by the dietary and Health Committee of experts in the United States showed that dietary imbalance is an important factor in a number of chronic diseases which has a high reference value to breast cancer, colon cancer and prostate cancer.The excess nutrition can also lead to obesity and increase the risk of type II diabetes.

Dunn ${ }^{[9]}$ and others have found a high incidence of colon cancer among immigrants from countries with low risk of colon cancer to Europe and the United States.It may be associated with a change in the structure of their diet after immigration. Study on Aune $^{[10]}$ found that dietary fiber intake of $10 \mathrm{~g}$ per day, the risk of colon cancer can be reduced by $10 \%$, intake of soy fiber, the risk of colon cancer dropped by $37 \%$ and three 
meals a day intake of whole grain fiber, the risk of colon cancer can be decreased by $17 \%$.

The obvious change of people's lifestyle and diet structure is also the main reason for the rising incidence of cardiovascular and cerebrovascular diseases. According to the Statistics Center of cardiovascular disease in our country, the number of our patients with cardiovascular disease is at least 230 million. There are 2 patients with cardiovascular disease among 10 adults, the annual cardiovascular death toll as high as 3 million. Cardiovascular disease has become the first major chronic disease with high mortality and high disability rate in china. Some scholars pointed out that only develop a good eating habit,can we hope to prevent the occurrence of cardiovascular and cerebrovascular diseases.

At present the incidence of gout is rising, especially the elderly are suffering from gout severely. The National Health Interview Survey (NHIS) datas show that the incidence of gout in the 45 to 65 age was $2.24 \%$, while the age group of 65 to 74 was $3.17 \%$. The gout has a close relationship with everyone's diet, long-term bad eating habits and an unbalanced diet will increase the incidence of gout, Xia Daozong summed up a variety of gout diet treatment program ${ }^{[11]}$.

\section{The Future Location of Chinese People's Eating Habits}

The future of Chinese people's eating habits should return to the traditional eating habits which are mainly rely on plant food, corn as staple food, adequate intake of vegetables and fruit while the animal food as a supplement. As a staple food grains are diverse, such as rice, sorghum, wheat, beans, corn and so on. There are steaming, boiling, stewing and other low-temperature cooking methods. The utility model has the advantages of no loss of nutrients, satisfying the need of surface sterilization, reducing the oxidation of oil and forming a variety of food varieties, such as rice, steamed bread, noodles, eight treasure porridge and the like ${ }^{[12]}$.Cereal is the main source of energy for the body, it can provide the carbohydrates, protein, dietary fiber and vitamins that the human body needs. The comprehensive nutrition and health care effect that grain provided make Chinese believe that eat grains can cure all diseases. People keep the "one day without meat, not a day without beans"and "light diet, vegetarian diet" principle. Formed a plant-based food, animal food as a supplement to the traditional Chinese diet. With the traditional diet of the Chinese nation wide polygamo not only conform to the organization structure of the human digestive tract, meet the nutrients required in the human body and contribute to human health, but also can shorten the human food chain and maximize the rational use of existing natural resources ${ }^{[15]}$. At the same time, the diet needs to grasp the proportion of animal food and vegetable food, staple food and non-staple food collocation, so that we can get comprehensive nutrition.There are many people who face the temptation to eat a single food and too full.In order to lose weight do not eat staple food is also not desirable. Regular diet, reasonable collocation and adjust the body balance can be healthy and get a long life.

\section{Summary}

In recent years, our country residents living conditions gradually superior and individual nutrition awareness has been greatly enhanced.But there are some people learning the Western diet culture phenomenon blindly, abandoned the traditional Chinese diet completely. It is easy to appear unbalanced nutritional situation, leading to 
the rise in the incidence of many chronic diseases. So a healthy diet should be the problem that everyone concerned.There is no unhealthy food, only unhealthy eating habits. We should restore the traditional Chinese food habit which is based on plant food and supplemented by animal food. Eat more fruits and vegetables, reducing the intake of salt and fat, making collocation of food reasonably according to personal taste, so that we can achieve a healthy diet.Make the healthy diet as a basic national policy, restore the traditional Chinese diet for the purpose, make scientific nutrition education and introduce specific policies to guide residents to eat healthy, is the urgent action of our government.

\section{Acknowledgement}

Zhongyuan Scholar (162101510003),Henan Province excellent science and technology innovation team (TCJ2014-391).

\section{References}

[1] Wang Xiaogeng.The relationship between the structure of dietary nutrition and the incidence of disease[J].Chinese Journal of medicine and Philosophy (A), 2013, (05): 38-39.

[2] Han Zhe.The relationship between climate change and disease[J].The latest medical information abstracts in world, 2015, (65): 97.

[3] Liu Yong,XiaoWei,Qin Zhenxian, Xiao Peigen.The interpretation and significance of"The medicine and food homology" [J].China modern Chinese medicine, 2015, (12): $1250-1252+1279$.

[4] Wang Tianhong, Zhang Yi, Liang Weiwen, Wang Zhang, Cui Zhengzhi. Study on the relationship between food efficacy and physical types under the guidance of health concept in East Medicine[J].World science and Technology Chinese medicine modernization, 2014, (01): 116-122.

[5] Zhai Fengying, Wang Huijun, Du Shufa, he Yu Na, Wang Zhihong, BM Popkin. Changes of dietary structure and nutritional status of Chinese residents [J]. urnal of medical research, 2006, (04): 3-6.

[6] Zhai Fengying, Wang Huijun, Wang Zhihong,He Yu Na, Du Shufa, Li Jie ,Yu Wentao. Changes and policy suggestions of dietary nutrition status of Chinese residents [J]. Food and nutrition in China, 2006, (05): 4-6.

[7] Chai Peipei, Zhang Yuhui, Wan Quan, Wang Xiufeng, Fang Li Ming, Zhang Mei. Study on the economic burden of disease in elderly malnutrition in China [J]. Chinese Journal of health economics, 2016, (03): 13-16.

[8] Li Lin, Wang Shuo, KLY. Study on the relationship between dietary pattern and phlegm dampness syndrome of coronary heart disease $[\mathrm{J}]$.Chinese Journal of traditional Chinese medicine, 2014, (05):1019-1020.

[9] Dunn JE. Cancer epidemiology in populations of the United States-with emphasis on Hawaii and California-and Japan[J].Cancer research,1975,35( 11 Pt.2):3240-3245. 
[10] Auen D, Chan DSM, Lau R, et al. Dietary fibre, whole grains, and risk of colorectal cancer[J].systematic review and dose-response me-ta-analysis of prospective studies.Brit Med J.2011,343:d6617.

[11] Xia Daozong,Zhong Yiping. Research Progress on nutrition and diet therapy of gout [J]. Journal of Zhejiang Chinese Medicine University, 2012, (11):1249-1252.

[12] Yan Maohua. Thinking from the western food culture to the traditional Oriental $\operatorname{diet}[J]$. Anhui Agricultural Sciences, 2014, (23):7996-7999.

[13] Zhao Lin, Bao Shanfen. The characteristics and advantages of Chinese traditional dietary structure [J]. Chinese Journal of food science,2004, (04):4-9. 